\title{
Expressions and relationship of Krüppel-like factor 15 and endothelial nitric oxide synthase in experimental deep venous thrombosis
}

\author{
Xianguang Yang", Yaoyu Xiang", Fuke Wang, Guofeng Cai, Yanlin Li, Ling Zhong, Li Pu, Yang Yang, \\ En Song \\ Department of Sports Medicine, the First Affiliated Hospital of Kunming Medical University, Kunming, China \\ Contributions: (I) Conception and design: E Song; (II) Administrative support: Y Li, G Cai; (III) Provision of study materials or patients: X Yang, F \\ Wang, L Zhong; (IV) Collection and assembly of data: Y Xiang, L Pu, Y Yang; (V) Data analysis and interpretation: E Song, Y Xiang, X Yang; (VI) \\ Manuscript writing: All authors; (VII) Final approval of manuscript: All authors. \\ \#These authors contributed equally to this work. \\ Correspondence to: En Song. Department of Sports Medicine, the First Affiliated Hospital of Kunming Medical University, No, 295 Xichang Road, \\ Kunming, China. Email: 641634233@qq.com.
}

Background: Deep vein thrombosis (DVT) is an early postoperative complication. Thrombosis formation, which is potentially life-threatening, seriously affects the rehabilitation of patients after surgery. We aimed to establish a C57 mouse model of DV'T and to examine the changes in the expression of Krüppel-like factor 15 (KLF15) and endothelial nitric oxide synthase (eNOS) in venous wall tissues, and we also investigated the regulatory relationship of KLF15 and eNOS in the thrombin-induced human umbilical vein endothelial cell (HUVEC) injury cell model.

Methods: The DVT model was established using the inferior vena cava (IVC) stenosis method. The expression levels of KLF15 and eNOS were analyzed using quantitative reverse transcription-polymerase chain reaction (qRT-PCR). In cell experiments, the expression of KLF15 and eNOS was analyzed in the model of thrombin-induced HUVEC injury with KLF15 siRNA.

Results: Compared to the control and sham-operated groups, KLF15 in the DVT group was upregulated, while eNOS was downregulated. The results of cell experiments revealed that KLF15 was downregulated in the thrombin+KLF15 siRNA group compared with the thrombin group. Meanwhile, eNOS was upregulated in the thrombin+KLF15 siRNA group compared with the thrombin group. These findings suggested that KLF15 regulated the expression of eNOS in the DVT model.

Conclusions: We successfully constructed a DVT mouse model. In the early stage of DVT formation, KLF15 regulated the expression and inhibited the antithrombotic effect of eNOS, resulting in thrombi formation.

Keywords: Venous thrombosis; gene expression regulation; human umbilical vein endothelial cells (HUVECs); Krüppel-like transcription factors; nitric oxide synthase type III

Submitted Jul 16, 2020. Accepted for publication Aug 28, 2020.

doi: $10.21037 / \mathrm{atm}-20-5828$

View this article at: http://dx.doi.org/10.21037/atm-20-5828 


\section{Introduction}

Venous thromboembolism (VTE), which includes deep vein thrombosis (DVT) and pulmonary thromboembolism (PTE), is the formation of blood plaques in veins (1). DVT is a venous reflux disorder that often occurs after major orthopedic surgery (2). It occurs when blood abnormally coagulates in a vein, causing complete or partial obstruction of the blood vessel. Although DVT can occur in various parts of the body, the deep veins of the lower extremities are most at risk. The thrombus formed by DVT can fall off and travel into the lung with the circulating blood, resulting in the pulmonary artery or its branches becoming obstructed. Such obstruction leads to pulmonary circulation disturbance, respiratory dysfunction, and eventually PTE, PTE making it one of the main causes of orthopedic perioperative death (3). Post-thrombosis syndrome (PTS) refers to complications that take place during the chronic phase of DVT. Venous reflux disorder and venous reverse flow can result in venous hypertension of the lower extremities, as well as swelling in the affected limb and chronic ulcers, which seriously affects the quality of life of patients (4).

Compared to other species, humans are prone to spontaneous DVT. In 1846, Virchow simulated human thrombosis using a canine model and proposed the classic triad of venous thrombosis: venous wall injury, hypercoagulable state, and blood stasis. In studies on DVT, thrombus samples generally cannot be obtained directly from the human body. Therefore, animal models that simulate the formation of DVT have been developed. Animal models of experimental venous thrombosis have been used for more than 50 years, and to date, 18 different animals have been used to establish DVT models (5). Establishing a simple, easy-to-build, stable, reproducible, and reliable DVT animal model is fundamental to in vivo research of the pathogenic mechanisms and pathological changes of DVT, as well as the evaluation of drug treatment. The inferior vena cava (IVC) stenosis-induced DVT mouse model avoids direct injury to venous endothelial cells, is consistent with the DVT process, and simulates the blood stasis state during venous thrombosis. Therefore, in the present study, the stenosis method was selected for the construction of the model. According to Brill et al.'s study (6), in which an IVC stenosis-induced DVT mouse model was established using a 30-G needle, the blood flow in the IVC was reduced by $90 \%$. However, that study did not take into account the specific operation of the experiment, degree of stenosis, thrombus formation status, and general condition of the mice. Therefore, the present study aimed to establish a mouse model of IVC stenosis-induced DVT via injection using a $30-\mathrm{G}$ needle, and to report a number of indices, including the length and weight of the thrombus, and the rates of stenosis, mortality, and thrombosis.

In the field of cardiovascular disease research, thrombin, a stimulating factor of coagulation/anticoagulation and inflammation, has been widely used to investigate mechanisms at the cellular level. Thrombin has been added to human umbilical vein endothelial cells (HUVECs) and human microvascular endothelial cells (HMVECs) at different concentrations and time points to investigate the mechanisms of action of genes and proteins, signaling pathways, and intercellular interactions, such as adhesion and migration (7). To explore the specific changes and regulatory mechanisms of DVT at the molecular level, it is necessary to simulate venous endothelial cell injury, as the key factor for DVT formation, and then analyze the mutual regulatory relationships between genes and proteins. Thus, establishing a model of venous endothelial cell injury is especially important.

Thrombin affects the coagulation/anticoagulation system and exhibits pro-inflammatory characteristics. It can induce the activation of venous endothelial cells. Compared to endothelial cell injury models induced by interleukin (IL)$1 \beta$ and tumor necrosis factor (TNF) $\alpha$, thrombin facilitates a better simulation of the changes in the thrombotic microenvironment. Therefore, in the present study, thrombin was chosen to stimulate HUVECs.

Krüppel-like factors (KLFs) are a class of transcriptional regulatory factors that are widely found in eukaryotes. KLFs are involved in various physiological and pathological processes, such as cell differentiation, cell proliferation, apoptosis, and tumorigenesis. Furthermore, KLFs are closely associated with cardiovascular diseases, such as myocardial hypertrophy, atherosclerosis, and coronary heart disease (8). The Krüppel-like zinc finger factor family comprises 17 members, named KLF1-17, according to the order in which they were discovered. KLFs can act as either transcriptional activators or repressors, with some possessing both functions (9). The transcriptional regulatory functions of KLFs depend on the specific activation or inhibition of their non-DNA-binding domains. Although a small number of studies have been performed on KLF15 in cardiovascular disease, no domestic or international reports currently exist on KLF15 in thrombosis, including 
arteriovenous thrombosis. Therefore, KLF15 was the KLF family member selected for the present study.

Endothelial nitric oxide synthase (eNOS) catalyzes the production of nitric oxide (NO) from L-arginine. eNOS is a key rate-limiting enzyme involved in the synthesis of NO. An important bioactive factor in organisms, NO dilates blood vessels, regulates blood pressure, inhibits platelet aggregation and adhesion, and inhibits the proliferation of vascular smooth muscle cells. NO also participates in the development and progression of various cardiovascular diseases, including hypertension, atherosclerosis, and pulmonary heart disease (10). However, the relationship between KLF15 and eNOS in DVT has yet to be reported.

Here, we investigated the association between KLF15 and eNOS in vivo and in vitro experiments. The present study aimed: (I) to establish a C57 mouse model of IVC stenosis-induced DVT, and summarize operation skills, and precautions during model construction; (II) to examine the expression of KLF15 and eNOS in the venous wall tissues of the model mice and analyze the relationship between the expression levels of KLF15 and eNOS under DVT; and (III) to explore the regulatory relationship between KLF15 and eNOS in a thrombin-induced HUVEC injury model in order to analyze the possible role of HUVECs in DVT formation.

We present the following article in accordance with the ARRIVE reporting checklist (available at http://dx.doi. org/10.21037/atm-20-5828).

\section{Methods}

\section{Experimental objects and grouping}

The present study involved 120 specific-pathogen-free (SPF) C57 mice (female; weight, $25 \pm 2 \mathrm{~g}$ ) obtained from the Medical Animal Experimental Center of Kunming Medical University, China [animal production license number: SCXK (Dian) 2011-0004]. During the experiment, the animals were handled according to the "Guiding Opinions on Treating Experimental Animals", issued by the Ministry of Science and Technology of China in 2006. The mice were raised under controlled temperature $\left(23 \pm 1^{\circ} \mathrm{C}\right)$ and humidity $(50 \% \pm 5 \%)$ conditions with $12 \mathrm{~h}$ light/dark cycle. The animals were acclimated to the housing conditions for 7 days before the experiments. The health and behavior of each group of mice were observed daily. The mice were randomly divided into three groups: the normal control group $(n=40)$, the sham-operated group $(n=40)$, and the
DVT group ( $\mathrm{n}=40)$. Experiments were performed under a project license (No.: KMMU2019089) granted by the Animal Experimental Ethical Committee of Kunming Medical University, in compliance with Kunming Medical University national or institutional guidelines for the care and use of animals.

\section{Establishment of the C57 mouse model}

To construct the model, the mice were anesthetized by intraperitoneal injection of $1 \%$ sodium pentobarbital at a dose of $50 \mathrm{mg} / \mathrm{kg}$. The mice in the sham-operated and DVT groups were subjected to the following surgical procedures.

\section{Sham-operated group}

Briefly, a $1.5-\mathrm{cm}$ incision was made along the midline of the abdomen, starting approximately $0.5 \mathrm{~cm}$ below the xiphoid process. Skin, layers of subcutaneous muscle, and the peritoneum were cut layer by layer, and the abdominal contents were gently exteriorized from the abdominal cavity to expose the IVC. The abdomen was then closed up, layer by layer; the muscles were closed with 5-0 absorbable suture, and the skin was closed with 5-0 silk suture. The mice were kept warm and fed a normal diet.

\section{DVT group}

The abdominal contents were gently exteriorized from the abdominal cavity, using the procedure described above. Then, gauze soaked with warm saline was placed above the peritoneal cavity of the mice. As shown in Figure 1A, mice were immobilized and disinfected. Gauze was also placed over the abdominal contents to maintain a constant temperature and prevent them from drying out (Figure 1B). At this point, the IVC was adequately exposed. The IVC was separated out at the triangular region below the junction of the left renal vein and the IVC with ophthalmic hooks used in the minimally invasive femtosecond procedure (Figure 1C,D). An electronic Vernier caliper was used to measure the diameter of the IVC (Figure 1E). After the measurement data had been recorded, a 7-0 prolene suture was passed behind the IVC (Figure $1 F$ ). Then, the assistant placed a $30-\mathrm{G}$ needle with a diameter of $0.30 \mathrm{~mm}$ on the surface of the IVC, parallel to the longitudinal axis (Figure 1G). Great care was taken to avoid damaging the IVC. The operator then ligated the IVC over the needle (Figure $1 H$ ), after which the $30-\mathrm{G}$ needle was promptly 

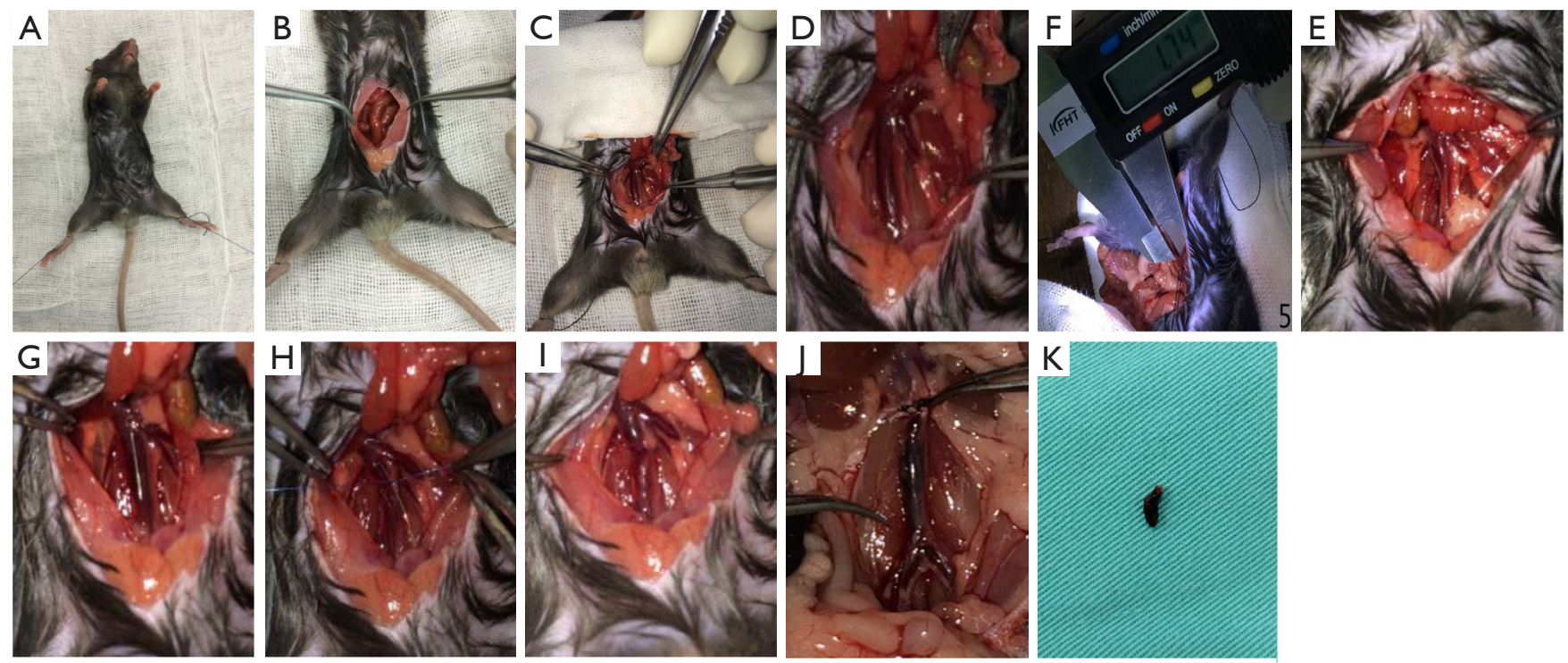

Figure 1 The process of building a deep vein thrombosis (DVT) model mouse. (A) The mice were immobilized and disinfected; (B) the abdomen was opened; (C,D) and the inferior vena cava (IVC) was exposed. (E) The diameter of the IVC was measured; (F) the blood vessels were separated, and a suture was passed behind the IVC. (G) A needle was placed on the IVC; $(\mathrm{H})$ a surgical knot was made; and then (I) the needle was removed. (J,K) The IVC thrombosis and thrombus tissue.

removed (Figure 1I). Subsequently, the blood flow to the IVC was reduced. To complete the model of IVC stenosis, all visible IVC branches needed to be ligated in the absence of endothelial cell damage. After the main operation, the mice were observed. Once the respiration and circulation of the mouse were confirmed to be stable, the abdomen was closed layer by layer. The abdominal muscles were closed with 5-0 absorbable suture, and the abdominal skin was sutured with 5-0 silk suture. The mice were kept warm and fed a normal diet.

During the process of constructing the model, the diameter of the mouse IVC was recorded at the site of stenosis. The cross-sectional area percentages of the residual lumen $\left(\pi r^{2} / \pi R^{2}\right)$ in the model mice of the DVT group were calculated based on the diameter of the needle (30 G, diameter $=0.30 \mathrm{~mm}$ ). After the model mice had fully awoke from anesthesia, their general condition was examined, including skin temperature, activity, swelling of the lower limbs, food intake, respiration, and heartbeat.

\section{Normal control group}

The mice were reared under normal conditions without any special treatment.

\section{Sample collection and data recording}

At 24 hours after the establishment of the model, specimens were collected. All of the mice were euthanized by overdose of sodium pentobarbital $(150 \mathrm{mg} / \mathrm{kg})$ via intraperitoneal injection. The abdomen of each mouse was disinfected, and surgical drapes were applied. The skin, subcutaneous tissues, and peritoneum of the C57 mice were incised layer by layer along the original incision line until the abdominal cavity was reached, and its contents were gently exteriorized. A gauze soaked with warm saline was placed above the peritoneal cavity of the mice. The abdominal contents were also covered with the gauze to maintain a constant temperature and to prevent them from drying out. The IVC was exposed between the left renal vein and the bifurcation of the common iliac vein. The modeled section of the IVC was examined, and the degree of swelling, color, and extent of local tissue reaction were recorded.

In the DVT group, tissues were collected from the thrombosed IVC, from between the site where the left renal vein joined the IVC and the bifurcation of the common iliac vein (Figure 17,K). Thrombus formation status was examined, and the lengths and total wet weights of the 
Table 1 The primer sequences of Krüppel-like factor 15 (KLF15), endothelial nitric oxide synthase (eNOS), and $\beta$-actin for qRT-PCR

\begin{tabular}{lcc}
\hline Genes & Sequences & Product size (bp) \\
\hline$\beta$-actin & $\mathrm{F}\left(5^{\prime}-3^{\prime}\right):$ ACGGCAAGTTCAACGGCACAG & 167 \\
& $\mathrm{R}\left(5^{\prime}-3^{\prime}\right):$ GACGCCAGTAGACTCCACGACA \\
KLF15 & $\mathrm{F}\left(5^{\prime}-3^{\prime}\right):$ TGGGTGATAGGCTGGTTGG \\
& $\mathrm{R}\left(5^{\prime}-3^{\prime}\right):$ AGAGGGCTTGAGAGTCGGG \\
eNOS & $\mathrm{F}\left(5^{\prime}-3^{\prime}\right):$ ATGTTGTCTGCGGCGATG \\
& $\mathrm{R}\left(5^{\prime}-3^{\prime}\right):$ GGTGCGTATGCGGCTTG & 117 \\
\hline
\end{tabular}

thrombi, as well as their corresponding vein walls, were measured. In the control group and the sham-operated group, $1.5-2.0 \mathrm{~cm}$ of vein wall tissues were collected from below the area where the left renal vein merged into the IVC.

\section{HUVEC culture and thrombin treatment}

HUVECs (CHI Scientific, Jiangyin, China) were cultured under standard conditions $\left(37{ }^{\circ} \mathrm{C}, 5 \% \mathrm{CO}_{2}\right)$ for three passages. The cells were then treated with $4 \mathrm{U} / \mathrm{mL}$ thrombin (BioVision, USA) for $0,4,8$, or 12 hours, and the cell samples were collected at the corresponding time points. In addition, HUVECs were treated with $0,2,4$, or $8 \mathrm{U} / \mathrm{mL}$ thrombin for 8 hours. Samples of HUVECs treated with different concentrations of thrombin were harvested at the corresponding time points. Total RNA was extracted from the samples, and the expression of KLF15 was analyzed by qRT-PCR.

\section{Cell transfection}

HUVECs were transfected with KLF15 siRNA (SANTA, USA). An appropriate mixing ratio (40 $\mu$ KLF15 siRNA and $10 \mu \mathrm{L}$ of transfection reagent) was selected. Serumfree medium and the interference fragment were added sequentially to a transfection tube and mixed together thoroughly. The transfection reagent was then added to the same tube. After thorough mixing, the mixture was placed at room temperature for 10-15 minutes. The media were aspirated from the culture plates. The cells were washed once with phosphate-buffered saline or serumfree medium, overlaid with the mixture, and incubated for 24 hours. Then, cell samples were collected. Four groups of HUVECs were prepared as follows: Control group: HUVECs were normally cultured and did not undergo any treatment. Cell samples were collected, and total RNA was extracted. Thrombin group: HUVECs were treated with thrombin at a concentration of $4 \mathrm{U} / \mathrm{mL}$ for 8 hours. Cell samples were then collected, and total RNA was extracted. KLF15 siRNA group: at 24 hours after transfection with KLF15 siRNA, cell samples were collected, and total RNA was extracted. KLF15 siRNA + thrombin group: At 16 hours after transfection with KLF15 siRNA, HUVECs were treated with $4 \mathrm{U} / \mathrm{mL}$ thrombin for 8 hours. Finally, cell samples were collected.

\section{qRT-PCR analysis}

RNA was extracted from cells and tissues with TRIZOL reagent (Invitrogen, USA). Complementary DNA ( $c D N A)$ was synthesized with the TAKARA reverse transcription synthesis kit (Fermentas, USA). The reverse transcription (RT) reaction was set up as follows: total RNA, $1 \mu \mathrm{g}$; oligo dT primer, $1 \mu \mathrm{L}$; RT mix, $1 \mu \mathrm{L}$; $5 \times$ reaction buffer, $4 \mu \mathrm{L}$; water, (nuclease-free), to $20 \mu \mathrm{L}$. The reaction was incubated at $37^{\circ} \mathrm{C}$ for 15 minutes in a PCR machine and then terminated by incubation at $85^{\circ} \mathrm{C}$ for $5 \mathrm{~s}$. The realtime PCR system was set up as follows: SYBR Green/ ROX qPCR Master Mix (2×), $10 \mu \mathrm{L}$; PCR forward primer (50 $\mu \mathrm{M}$, Sangon Biotech), $0.16 \mu \mathrm{L}$; PCR reverse primer (50 $\mu \mathrm{M}$, Sangon Biotech), $0.16 \mu \mathrm{L}$; template DNA, $5 \mu \mathrm{L}$; water (nuclease-free), to $20 \mu \mathrm{L}$. The solutions were mixed thoroughly, and the 96-well plate was covered with sealingfilm (Bio-Rad). The two-step real-time PCR amplification procedure was as follows: stage 1: initial denaturation, $95^{\circ} \mathrm{C}$ for $10 \mathrm{~min}$. Stage 2,40 cycles: denaturation, $95^{\circ} \mathrm{C}$ for $15 \mathrm{~s}$; annealing/extension, $60^{\circ} \mathrm{C}$ for $1 \mathrm{~min}$. The ABI Prism $7300 \mathrm{HT}$ system was used to perform real-time fluorescence-based quantitative PCR analysis. The $2^{-\Delta \Delta C t}$ method was used to analyze the results. The primers used are listed in Table 1. 


\section{Western blot analysis}

Proteins were extracted from cells and tissues with radioimmunoprecipitation assay (RIPA) lysis buffer (Beyotime, Shanghai, China). The BCA Protein Assay Kit (Beyotime, Shanghai, China) was employed to determine protein concentration. Total protein $(30 \mu \mathrm{g})$ was separated by $10 \%$ sodium dodecyl sulfatepolyacrylamide gel electrophoresis. Electrophoresis was carried out first at a voltage of $80 \mathrm{~V}$ (for the concentrating gel), and then at a voltage of $140 \mathrm{~V}$ (for the separation gel). After electrophoresis, the proteins were transferred to a polyvinylidene fluoride membrane (Millipore) using a wet transfer method. Subsequently, the membrane was incubated with blocking agent ( $5 \%$ skimmed milk) at room temperature for 1 hour. After blocking, the membrane was incubated first with KLF15 (SANTA, USA), eNOS (SANTA, USA), and -actin (ABclonal, Wuhan, China) antibodies, respectively [diluted 1:1,000 with Tris-buffered saline-Tween 20 (TBST) solution] at $4{ }^{\circ} \mathrm{C}$ overnight. Next, the membrane was incubated with secondary antibody (ABclonal, Wuhan, China) (diluted 1:5,000 with TBST) at RT for 1 hour, before washing. The ECL Plus chromogenic reagent (Bio-Rad, USA) was added to the membrane to visualize the proteins, and images were taken under a chemical imaging system (Bio-Rad). The grayscale values of the protein bands were determined in Image $2 \times$ software.

\section{Statistical analysis}

All measured values were subjected to statistical analysis using SPSS17.0 software (International Business Machine Company, New York, USA). All measurement data were expressed as mean \pm standard deviation (mean $\pm \mathrm{SD}$ ). Data were analyzed by one-way analysis of variance. $\mathrm{P}<0.05$ was considered to be statistically significant.

\section{Results}

\section{Construction of a mouse model of DVT}

No mice died in any group. The anesthesia onset time was approximately 5-20 minutes. The longest time interval from intraperitoneal injection to the appearance of active movement was approximately $60 \mathrm{~min}$ (average: $40 \mathrm{~min}$ ). The average operative time was $8-10 \mathrm{~min}$ in the sham-operated group and $20 \mathrm{~min}$ in the DVT group.
After awakening from anesthesia, the sham operated mice were able to eat and drink normally. Their breathing and heartbeats were also normal. After the surgery, the mice were observed for a 24-hour period, during which the mice showed normal movement of the lower extremities and normal skin temperature.

In the DVT group, the IVC became dilated and the blood stagnated below the stenotic site after the establishment of the model. However, the IVC still had blood flow and exhibited fair vascular elasticity. After awakening from anesthesia, the mice were able to eat and drink normally. Their breathing and heartbeats were also normal. After surgery, the mice were monitored for 24 hours. Swelling of the bilateral hind limbs was observed in 18 mice. These mice had noticeably restricted movement, and their skin temperature was decreased. Ten mice showed stiffness and immobility in both lower extremities.

In the normal control and sham-operated groups (Figure $2 A, B$ ), the IVC was examined by the naked eye when the specimens were collected, 24 hours after the establishment of the model. The diameter of the IVC had not obviously changed. Furthermore, the diameter of the blood vessel appeared uniform. The IVC was unobstructed, with a flow of dark-red blood. The blood vessel appeared elastic, and the vessel wall was soft. No dilatation of the abdominal mesenteric vein was observed. Venous wall tissues of $1.5-2.0 \mathrm{~cm}$ in length were collected below the junction of the left renal vein and the IVC (Figure 2). In the DVT group (Figure 2C), the specimens were collected 24 hours after the model was established. By the naked eye, the wall of the IVC was observed to have become black, and its elasticity was decreased. Moreover, black clot-like substances were found in the IVC, which compressed the blood vessel and did not move. Tissues were collected from the thrombosed IVC from between the site where the left renal vein joined the IVC and the bifurcation of the common iliac vein. The length and wet weight of the thrombus were measured.

The rates of mortality, thrombosis, and stenosis in the DVT group are shown in Table 2, along with the crosssectional area percentage of the residual lumen. Table 3 shows the body weight, diameter of the IVC, length of the thrombus, and wet weight of the thrombus in the DVT group.

\section{The expression status of KLF15 and eNOS in the DVT mice}

The KLF15 and eNOS mRNA expression in the venous 

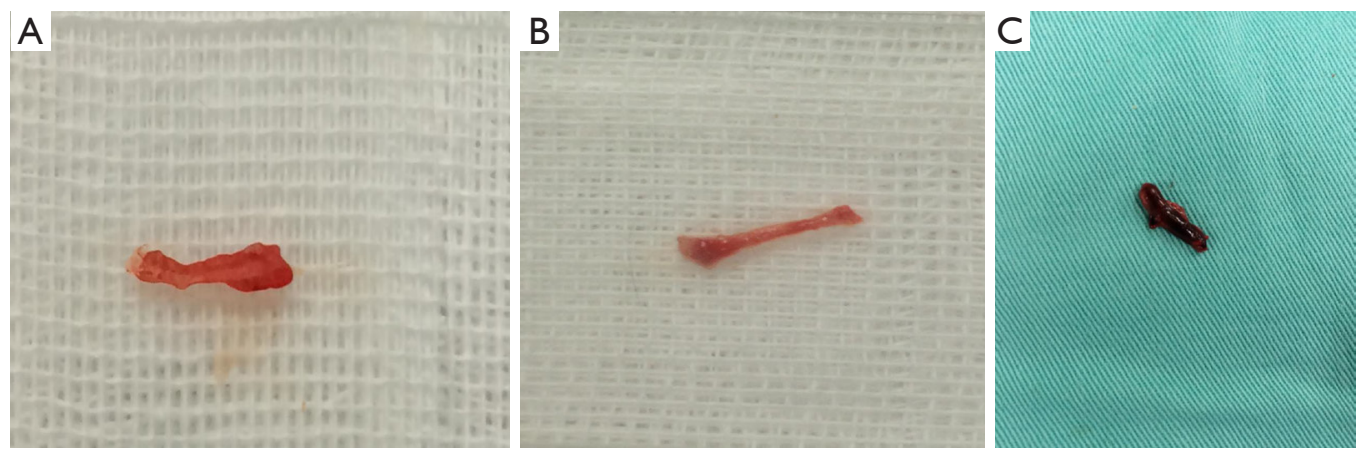

Figure 2 Photographs of blood vessels and thrombi. The venous walls collected from the mice in the normal control group (A) and the sham-operated group (B). The thrombotic tissue collected from the mice in the DVT group (C). DVT, deep vein thrombosis.

Table 2 The rates of thrombosis, mortality, and stenosis, and the cross-sectional area percentage of the residual lumen in the deep vein thrombosis (DVT) group

\begin{tabular}{ll}
\hline Parameters & Value \\
\hline Mortality rate & $0 \%$ \\
Thrombosis rate & $85.30 \%$ \\
Cross-sectional area percentage of the residual lumen & $9.2 \% \pm 0.8 \%$ \\
Stenosis rate & $90.8 \% \pm 0.8 \%$ \\
\hline
\end{tabular}

Table 3 Body weight, diameter of the inferior vena cava (IVC), length of the thrombus, and wet weight of the thrombus in the deep vein thrombosis (DVT) group

\begin{tabular}{ll}
\hline Parameters & Value \\
\hline Body weight $(\mathrm{g})$ & $25.2 \pm 1.3$ \\
Diameter of the IVC $(\mathrm{mm})$ & $0.99 \pm 0.04$ \\
Length of the thrombus $(\mathrm{mm})$ & $5.85 \pm 0.25$ \\
Weight of the thrombus $(\mathrm{g})$ & $0.27 \pm 0.02$ \\
\hline
\end{tabular}

walls of the mice were determined by qRT-PCR. The results showed that KLF15 was significantly upregulated in the DVT group in comparison with the normal control and the sham-operated groups (Figure $3 A$ ). In contrast, eNOS was significantly downregulated in the DVT group compared with the other two groups (Figure 3B). No statistically significant differences in the expression of KLF15 and eNOS existed between the normal control group and the sham-operated group (Figure 3). These results suggested that KLF15 may be involved in the development of DVT through the regulation of eNOS expression.

\section{Establishment of a model of thrombin-induced HUVEC}

HUVECs were treated with 0,2 , 4, or $8 \mathrm{U} / \mathrm{mL}$ thrombin for 8 hours. KLF15 was upregulated after treatment with 2 or $4 \mathrm{U} / \mathrm{mL}$ thrombin, but it was downregulated by thrombin at a concentration of $8 \mathrm{U} / \mathrm{mL}$ (Figure $4 A$ ). Following treatment with $4 \mathrm{U} / \mathrm{mL}$ thrombin, KLF15 expression peaked at a level significantly greater than that at other concentrations. Then, HUVECs were treated with $4 \mathrm{U} / \mathrm{mL}$ thrombin for 0,4 , 8 , or 12 hours. KLF15 was significantly upregulated at 4 and 8 hours (Figure $4 B$ ). Therefore, in the subsequent experiments, HUVECs 
A

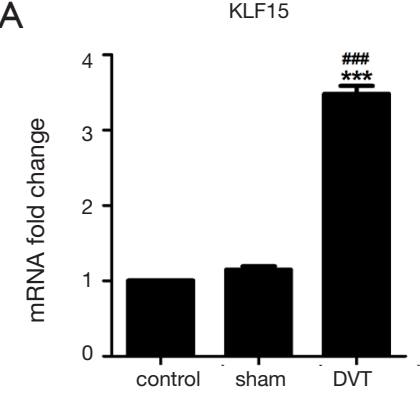

B eNos

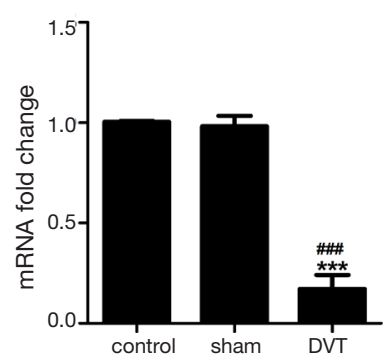

Figure 3 The expression of KLF15 and eNOS in blood vessels of DVT mice. (A) The mRNA expression of KLF15 in the three groups was detected by qRT-PCR assay. (B) The mRNA expression of eNOS in the three groups was detected by qRT-PCR assay. ${ }^{*} \mathrm{P}<0.05$, ${ }^{* *} \mathrm{P}<0.01$ and ${ }^{* * *} \mathrm{P}<0.001$ vs. the control group; ${ }^{\#} \mathrm{P}<0.05,{ }^{\# \#} \mathrm{P}<0.01$ and ${ }^{\# \# \#} \mathrm{P}<0.001$ vs. the sham group. KLF15, Krüppel-like factor 15 ; eNOS, endothelial nitric oxide synthase; DVT, deep vein thrombosis.
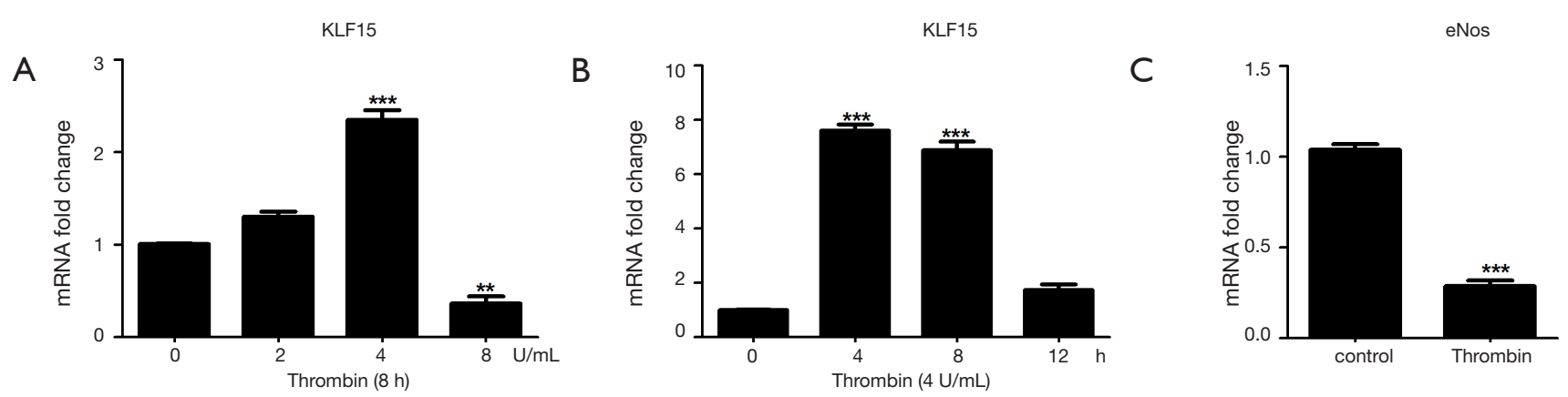

Figure 4 The effects of thrombin on the expression of KLF15 and eNOS mRNA in HUVECs. (A) HUVECs were treated with thrombin $(0,2$, 4, or $8 \mathrm{U} / \mathrm{mL})$ for 8 hours. (B) HUVECs were treated with $4 \mathrm{U} / \mathrm{mL}$ thrombin for 4, 8, or 12 hours. mRNA expression levels of KLF15 were investigated using qRT-PCR. (C) The mRNA expression levels of eNOS was investigated using $\mathrm{qRT}-\mathrm{PCR}$. ${ }^{*} \mathrm{P}<0.05$, ${ }^{*} \mathrm{P}<0.01$ and ${ }^{* * *} \mathrm{P}<0.001$ vs. control group. KLF15, Krüppel-like factor 15 ; eNOS, endothelial nitric oxide synthase; HUVEC, human umbilical vein endothelial cell.

were treated with thrombin at a concentration of $4 \mathrm{U} / \mathrm{mL}$, and the treatment duration was set to 8 hours. Then, the expression of eNOS was detected in a thrombin-induced HUVEC model using qRT-PCR. As shown in Figure $4 C$, the expression of eNOS was markedly suppressed in the thrombin-induced HUVEC model compared to in the control group (Figure 4C).

\section{The effect of KLF15 siRNA on the expression of eNOS in a thrombin-induced HUVEC model}

In the thrombin group, the mRNA and protein expression levels of KLF15 were upregulated, while those of eNOS were significantly downregulated compared to those in the control group. In contrast, the level of KLF15 expression was inhibited and that of eNOS was significantly promoted in the thrombin + KLF15 siRNA group compared with the thrombin group (Figure 5A). No significant differences were observed between the thrombin group and the thrombin + NC siRNA group (Figure 5). The results of western blot revealed the same change as did the qRT-PCR results (Figure 5B,C), suggesting that KLF15 siRNA can recover the reduction in eNOS expression induced by thrombin. Therefore, we speculated that KLF15 downregulated the expression of eNOS in our thrombin-induced HUVEC model.

\section{Discussion}

Mice have 30,000 genes, approximately $80 \%$ of which have a human counterpart. The similarities between human and mouse DNA sequences allow scientists to use mice to 

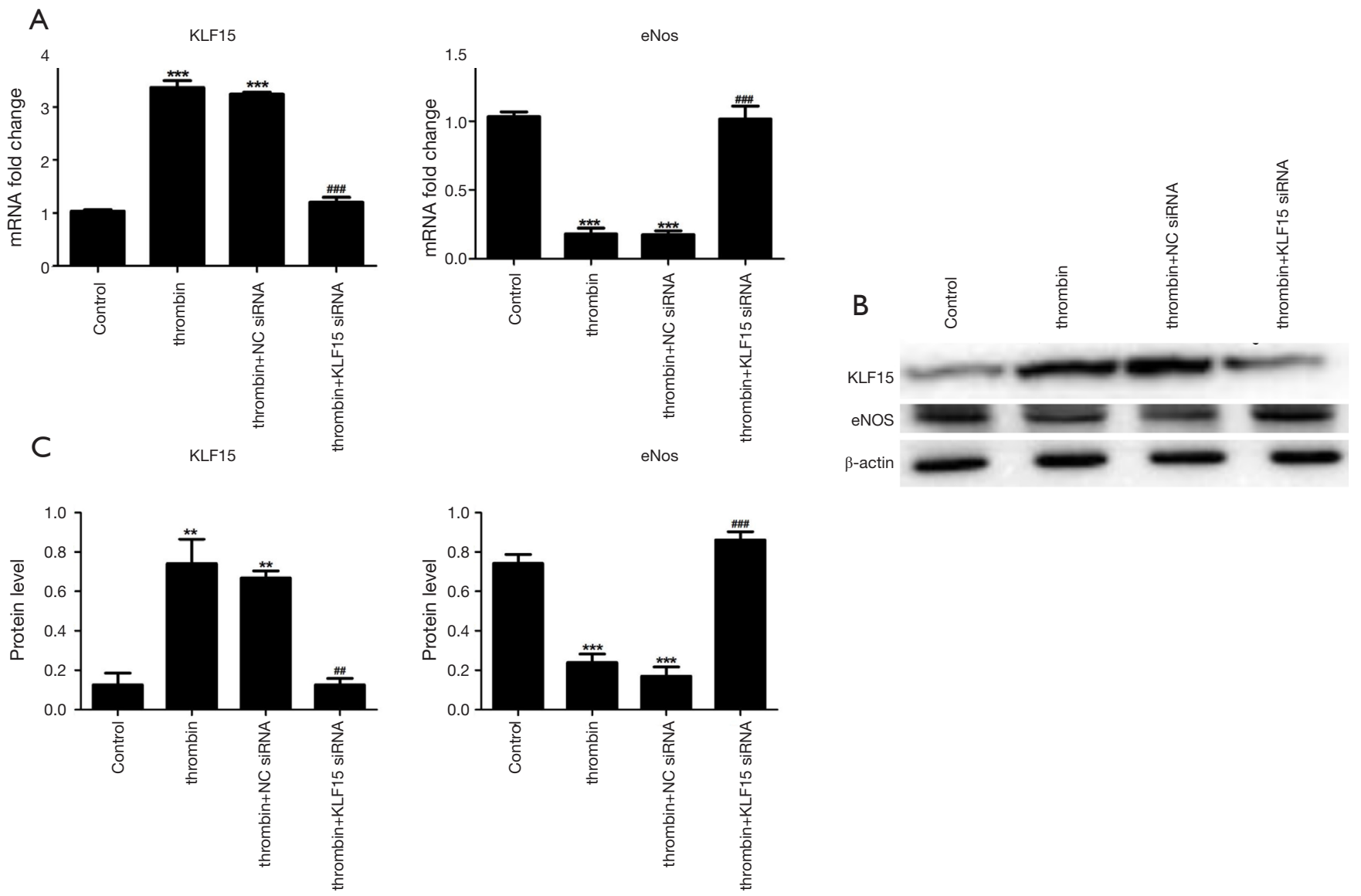

Figure 5 The effect of KLF15 siRNA on eNOS in a thrombin induced-HUVEC cell model. The mRNA and protein expression levels of KLF15 and eNOS in thrombin treated-HUVEC cells transfected with KLF15 siRNA and negative control were detected by qRT-PCR (A) and Western blot (B and $\mathrm{C}$ ). ${ }^{*} \mathrm{P}<0.05,{ }^{* *} \mathrm{P}<0.01$ and ${ }^{* * *} \mathrm{P}<0.001$ vs. the control group; ${ }^{*} \mathrm{P}<0.05,{ }^{\# \#} \mathrm{P}<0.01$ and ${ }^{\# \# \#} \mathrm{P}<0.001$ vs. the thrombin group. KLF15, Krüppel-like factor 15; eNOS, endothelial nitric oxide synthase; HUVEC, human umbilical vein endothelial cell.

study the expression characteristics of human genes. Mouse genome sequencing has made the physiological regulation of mouse genes, such as gene addition (transgenesis) and gene deletion (gene knockout), possible, which has greatly enhanced our understanding of the thrombotic process (11). By constructing a mouse DVT model using the IVC stenosis method, the direct mechanical injury to venous endothelial cells can be reduced and unobstructed blood flow in certain vessels under the environment of local venous blood stasis can be maintained, thereby simulating the clinical states of DVT. Such a model can be used to investigate the various stages of thrombosis, from thrombus formation and growth to partial thrombus regression and vascular recanalization (6). In the present study, a 30-G needle was used to construct the IVC stenosis-induced mouse model. In the DVT model group, the rates of thrombosis, mortality, and stenosis were $85.3 \%, 15 \%$, and $90.8 \% \pm 0.8 \%$, respectively. The cross-sectional area percentage of the residual lumen was $9.2 \% \pm 0.8 \%$, and the diameter of the IVC was $0.99 \pm 0.04 \mathrm{~mm}$. The thrombus measured $5.85 \pm 0.25 \mathrm{~mm}$ in length and had a wet weight of $0.27 \pm 0.02 \mathrm{~g}$. A complete thrombus was formed within 24 hours of the model being constructed. Therefore, this DVT model was stable and reliable.

The operational skills and precautions of anesthesia can be summarized as follows: during anesthesia, the needle was inserted into the abdomen of the mice, avoiding the midline. The needle was inserted at an angle of $45^{\circ}$ to the abdomen, in the direction of the tail to the head. The needle was used to penetrate the abdominal wall, and the anesthetic was injected. Failure to insert the needle deep enough could lead to the anesthetic being injected into the 
abdominal wall, resulting in the anesthetic effect being lost or delayed. However, inserting the needle too deeply could puncture the intestine, bladder, or important blood vessels (the IVC and its branches or the abdominal aorta and its branches), causing the death of the mouse. Furthermore, inserting the needle into an inappropriate site could result in the anesthetic being injected into the perirenal tissues, leading to perinephric hematoma. The occurrence of perinephric hematoma would affect the surgical operation and cause a poor anesthetic effect. By paying close attention to the above aspects, the adverse consequences of improper anesthesia may be reduced. Inadequate anesthesia is not conducive to surgical operation, while excessive anesthesia may cause death.

The operational skills and precautions during the construction of the model are as follows: During the operation, we noticed the presence of mesenteric white adipose tissue visible to the naked eye in the triangular area below the junction of the left renal vein and the IVC. The IVC could be easily separated from the abdominal aorta in this area. Therefore, this area was selected as an important marker point for the separation of the IVC in the construction of the model. Due to the existence and distributional variation of the posterior branch of the IVC, careless operation might tear the posterior branch when separating the IVC from the abdominal aorta, resulting in bleeding and death. Therefore, the IVC should be separated gently and carefully. Before the operation, the ends of the needle should be polished as smoothly as possible. After the surgeon had ligated the IVC in the presence of the needle, the needle was pulled out gently and parallel to the IVC to avoid scratching the blood vessel wall, which might cause bleeding. After model construction, the body temperature of the mice can drop due to blood loss and anesthesia, resulting in death. Therefore, postoperative heat preservation measures, such as using a warm air blower, should be stepped up. This step is critical. In the preliminary experiment, hypothermia might lead to significantly increased mortality in the mice, despite successful anesthesia and model construction. The duration of the operation (from the opening to the closure of the abdomen) should be confined to 20-25 minutes to help reduce the mortality rate.

KLF15, a member of the Kruppel-like factor family, has three highly conserved zinc finger structures (12). These zinc finger structures contain nuclear localization signals, which allow the translocation of KLF15 into the nucleus and the localization of KLF15 to the CACCC element and GC-rich regions of the DNA. Zinc fingers 2 and 3 are essential for the nuclear localization of KLF15 (13). KLF15 is widely expressed in tissues and organs, especially in the heart, kidney, liver, adipocytes, and skeletal muscles (14). KLF15 plays a key role in numerous biological processes, including cell proliferation, differentiation, development, and apoptosis (12). KLF15 expression is regulated by many substances; activated glucocorticoid receptor, glucocorticoid signals, and fasting upregulate KLF15 expression, while feeding, obesity, and interleukin (IL)-17 inhibit KLF15 expression (15). KLF15 has crucial functions in multiple processes, including in heart diseases, in kidney diseases, glucose metabolism, and energy utilization (16-18). KLF15 can significantly inhibit cardiac hypertrophy induced by Ang II (13). Lin et al. (19) found that KLF2 can bind to the promoter of and induce the expression of thrombomodulin (TM), and stimulate the exchange of protein $\mathrm{C}$ and activated protein $\mathrm{C}$ to inhibit thrombus formation. At the same time, KLF2 over-expression can inhibit the expression of plasminogen activator inhibitor 1 (PAI-1) and tissue factor (TF) induced by cytokines, causing an antithrombotic effect. However, no studies on the role of KLF15 in DVT currently exist.

In our study, qRT-PCR was employed to determine the mRNA expressions of KLF15 and eNOS in the venous walls of normal control, sham-operated, and DVT model mice. KLF15 was markedly upregulated in the DVT group compared with the normal control and sham-operated groups, while the expression of eNOS was downregulated in the DVT group compared with the normal control and sham-operated groups. No statistically significant differences were observed in the expression of the two genes between the normal control group and the shamoperated group. These findings suggested that KLF15 has an important role in process of DVT, with overexpression of KLF15 downregulating eNOS expression to promote thrombus formation. However, little research to date has explored the regulatory relationship between KLF15 and eNOS.

Blood coagulation includes three important steps: the formation of prothrombin activator, the formation of thrombin, and the formation of fibrin. Of these processes, the formation of thrombin is essential. Thrombin, a serine protease, promotes clot formation by converting fibrinogen into fibrin and is also involved in various vascular biological reactions, including cell regulation, platelet aggregation and secretion, endothelial cell activation, and inflammation. Thrombin links tissue damage to blood coagulation and 
inflammatory reactions, and even participates in immune responses. Thrombin induces vascular endothelial cells to undergo morphological changes, and the permeability of the vascular endothelial monolayer to increase, leading to local plasma protein leakage and edema. The altered microvascular permeability of organs changes the internal environment and causes a series of pathological changes (20). The stimulation of vascular endothelial cells with thrombin induces a series of reactions, including increased vascular permeability to plasma proteins and the release of soluble mediators such as plateletactivating factor, IL-8, vascular endothelial growth factor, and matrix metalloproteinases (21). The addition of thrombin to HUVECs activates the protease-activated receptors 1 and 4 , upregulating the downstream inflammation-related factors and cell adhesion factors (such as vascular cell adhesion molecule 1 , intercellular adhesion molecule 1 , and E-selectin) and promoting the adhesion of leukocytes to the venous endothelial cells. These effects are mainly mediated through the nuclear factor kappa B signaling pathway (22).

In the present study, HUVECs were treated with various concentrations of thrombin $(0,2,4$, or $8 \mathrm{U} / \mathrm{mL})$ for 8 hours. Thrombin at a concentration of $4 \mathrm{U} / \mathrm{mL}$ had the strongest effect on KLF15 and was subsequently used to treat HUVECs for various lengths of time $(0,4,8$, and 12 hours), which revealed that the best treatment duration was 8 hours. HUVECs were transfected with KLF15 siRNA to inhibit the expression of KLF15, and the expression of KLF15 and eNOS was examined at the mRNA and protein levels. We found that KLF15 was downregulated in the thrombin+KLF15 siRNA group compared with the thrombin group, while eNOS was upregulated in the thrombin+KLF15 siRNA group compared with the thrombin group. KLF15 and eNOS exhibited opposite expression trends, suggesting that KLF15 could regulate eNOS expression in the thrombin-treated HUVEC cell model. SenBanerjee et al. (23) reported that KLF2 can induce the expression of eNOS, which is an important enzyme for vascular tone regulation and thrombosis prevention. KLF15 overexpression has been reported to markedly enhance cell viability and the quantity of released NO in TNF- $\alpha$-induced Eahy926 cells, as well as to increase the expression levels of eNOS (24).

In the early stage of DVT, coagulation and anticoagulation mechanisms exist simultaneously and are in a state of dynamic change. Once the balance between procoagulation and anticoagulation is broken, a stronger procoagulant action may lead to thrombus formation, while a stronger anticoagulant effect inhibits thrombus formation.
Based on our animal and cell experiments, we speculate that KLF15 becomes activated in the first stage of DVT. KLF15 may downregulate eNOS expression, leading to a decrease in the eNOS-mediated NO production and reduced NO biological activity. The inhibitory effects of $\mathrm{NO}$ on the expression of endothelial cell adhesion factor, the activation and aggregation of platelets, and the secretion and expression of inflammatory factors were lost, resulting in thrombus formation.

In conclusion, DVT is affected by multiple factors. The mechanisms and network-regulatory relationships underlying DVT are complex. Our hypothesis that KLF15 acts as a regulator of eNOS expression provides a novel idea for future investigations of the molecular mechanism of DVT. The activation of KLF15 in the early stage of DVT and the related mechanisms, as well as the mechanisms by which KLF15 regulates eNOS and the related signaling pathways, still need to be elucidated. Further research on gene-knockout mice and the related signal transduction pathways in DVT is needed.

\section{Acknowledgments}

Funding: This study was supported by the Surface Project of Yunnan Applied Basic Research Program (2017FB110).

\section{Footnote}

Reporting Checklist: The authors have completed the ARRIVE reporting checklist. Available at http://dx.doi.org/10.21037/ atm-20-5828

Data Sharing Statement: Available at http://dx.doi. org/10.21037/atm-20-5828

Conflicts of Interest: All authors have completed the ICMJE uniform disclosure form (available at http://dx.doi. org/10.21037/atm-20-5828). The authors have no conflicts of interest to declare.

Ethical Statement: The authors are accountable for all aspects of the work in ensuring that questions related to the accuracy or integrity of any part of the work are appropriately investigated and resolved. Experiments were performed under a project license (No.: KMMU2019089) granted by the Animal Experimental Ethical Committee of Kunming Medical University, in compliance with Kunming Medical University national or institutional guidelines for 
the care and use of animals.

Open Access Statement: This is an Open Access article distributed in accordance with the Creative Commons Attribution-NonCommercial-NoDerivs 4.0 International License (CC BY-NC-ND 4.0), which permits the noncommercial replication and distribution of the article with the strict proviso that no changes or edits are made and the original work is properly cited (including links to both the formal publication through the relevant DOI and the license). See: https://creativecommons.org/licenses/by-nc-nd/4.0/.

\section{References}

1. Tenna AM, Kappadath S, Stansby G. Diagnostic tests and strategies in venous thromboembolism. Phlebology 2012;27 Suppl 2:43-52.

2. Wadajkar AS, Santimano S, Rahimi M, et al. Deep vein thrombosis: current status and nanotechnology advances. Biotechnol Adv 2013;31:504-13.

3. Gudipati S, Fragkakis EM, Ciriello V, et al. A cohort study on the incidence and outcome of pulmonary embolism in trauma and orthopedic patients. BMC Med 2014;12:39.

4. Cosmi B, Palareti G. "Early thrombus removal" in iliacfemoral deep vein thrombosis for prevention of postthrombotic syndrome. Ann Transl Med 2019;7:S343..

5. Levi M, Dorffle-Melly J, Johnson GJ, et al. Usefulness and limitations of animal models of venous thrombosis. Thromb Haemost 2001;86:1331-3.

6. Brill A, Fuchs TA, Chauhan AK, et al. von Willebrand factor-mediated platelet adhesion is critical for deep vein thrombosis in mouse models. Blood 2011;117:1400-7.

7. Huntington JA. Molecular recognition mechanisms of thrombin. J Thromb Haemost 2005;3:1861-72.

8. McConnell BB, Yang VW. Mammalian Kruppellike factors in health and diseases. Physiol Rev 2010;90:1337-81.

9. Pei J, Grishin NV. A new family of predicted Kruppel-like factor genes and pseudogenes in placental mammals. PLoS One 2013;8:e81109.

10. Toda N, Tanabe S, Nakanishi S. Nitric oxide-mediated coronary flow regulation in patients with coronary artery disease: recent advances. Int J Angiol 2011;20:121-34.

11. Diaz JA, Obi AT, Myers DD Jr, et al. Critical review of mouse models of venous thrombosis. Arterioscler Thromb Vasc Biol 2012;32:556-62.

12. Pearson R, Fleetwood J, Eaton S, et al. Kruppel-like transcription factors: a functional family. Int J Biochem
Cell Biol 2008;40:1996-2001.

13. Leenders JJ, Wijnen WJ, van der Made I, et al. Repression of cardiac hypertrophy by KLF15: underlying mechanisms and therapeutic implications. PLoS One 2012;7:e36754.

14. Mori T, Sakaue H, Iguchi H, et al. Role of Kruppellike factor 15 (KLF15) in transcriptional regulation of adipogenesis. J Biol Chem 2005;280:12867-75.

15. Ahmed M, Gaffen SL. IL-17 inhibits adipogenesis in part via C/EBPalpha, PPARgamma and Kruppel-like factors. Cytokine 2013;61:898-905.

16. Takashima M, Ogawa W, Hayashi K, et al. Role of KLF15 in regulation of hepatic gluconeogenesis and metformin action. Diabetes 2010;59:1608-15.

17. Gray S, Feinberg MW, Hull S, et al. The Kruppel-like factor KLF15 regulates the insulin-sensitive glucose transporter GLUT4. J Biol Chem 2002;277:34322-8.

18. Fisch S, Gray S, Heymans S, et al. Kruppel-like factor 15 is a regulator of cardiomyocyte hypertrophy. Proc Natl Acad Sci U S A 2007;104:7074-9.

19. Lin Z, Kumar A, SenBanerjee S, et al. Kruppel-like factor 2 (KLF2) regulates endothelial thrombotic function. Circ Res 2005;96:e48-57.

20. Krisinger MJ, Goebeler V, Lu Z, et al. Thrombin generates previously unidentified $\mathrm{C} 5$ products that support the terminal complement activation pathway. Blood 2012;120:1717-25.

21. Xue M, Hollenberg MD, Yong VW. Combination of thrombin and matrix metalloproteinase-9 exacerbates neurotoxicity in cell culture and intracerebral hemorrhage in mice. J Neurosci 2006;26:10281-91.

22. Alabanza LM, Bynoe MS. Thrombin induces an inflammatory phenotype in a human brain endothelial cell line. J Neuroimmunol 2012;245:48-55.

23. SenBanerjee S, Lin Z, Atkins GB, et al. KLF2 Is a novel transcriptional regulator of endothelial proinflammatory activation. J Exp Med 2004;199:1305-15.

24. Liu B, Xu L, Yu X, et al. Protective effect of KLF15 on vascular endothelial dysfunction induced by TNFalpha. Mol Med Rep 2018;18:1987-94.

(English Language Editor: J. Reynolds)

Cite this article as: Yang X, Xiang Y, Wang F, Cai G, Li Y, Zhong L, Pu L, Yang Y, Song E. Expressions and relationship of Krüppel-like factor 15 and endothelial nitric oxide synthase in experimental deep venous thrombosis. Ann Transl Med 2020;8(17):1090. doi: 10.21037/atm-20-5828 